\title{
MATHEMATICAL MODEL AND EXPERIMENTAL PROCEEDINGS TO DETERMINE ROLL WAVES IN OPEN CHANNELS
}

\author{
G. H. Fiorot ${ }^{\mathrm{a}, \mathrm{b}}$, \\ G. F. Maciel ${ }^{\mathrm{a}, \mathrm{c}}$, \\ and C. Kitano ${ }^{\text {a,d }}$ \\ ${ }^{a}$ Universidade Estadual Paulista "Júlio de \\ Mesquita Filho" - Campus de Ilha Solteira \\ CEP 15385-000, Ilha Solteira, São Paulo, \\ Brasil \\ ${ }^{b}$ Departamento de Engenharia Mecânica \\ Avenida Brasil, 56 \\ ghfiorot@aluno.feis.unesp.br \\ ${ }^{\mathrm{c}}$ Departamento de Engenharia Civil \\ Alameda Bahia, 550 \\ maciel@dec.feis.unesp.br \\ ${ }^{\mathrm{d}}$ Departamento de Engenharia Elétrica \\ Campus III \\ kitano@dee.feis.unesp.br \\ ABSTRACT \\ The goal of this paper is consolidate a representative model previously \\ developed by RMVP team (Rheological Studies on Viscous and Viscous- \\ plastic Materials) from UNESP - Ilha Solteira, for a typical phenomenon \\ that occurs on spillways, river's bed, landslides, mudflows, blood flows, for \\ Newtonian and non-Newtonian fluids, known as roll waves. Another goal of \\ this paper is present an experimental project designed for capturing \\ measurements (amplitude and wavelength) of these instabilities. From a \\ mathematical perspective, a first-order analytical model is showed, based on \\ Cauchy's equations system, once developed by the team (Ferreira, 2007), \\ which provides a generation condition for roll waves through temporal \\ linear stability analysis. This model follows the remarkable work of \\ Dressler (1949) and it is able to generate roll waves for many rheological \\ configurations, from Newtonian to Herschel \& Bulkley models, \\ representing clean water up to muddy mixtures, respectively. A numerical \\ routine developed in Matlab/Simulink is used to show some results that \\ illustrate roll waves pattern. Due to the lack of roll waves data (amplitude \\ and wavelength), the team started to focus on the experimental approach of \\ the phenomenon, aiming to design an apparatus that would be capable to \\ reproduce roll waves in special conditions of flow, isolated from external \\ perturbations. This project is here presented along with a proposal of a \\ photometric system to ascertain measures of the flow height through light \\ absorption technique, based on experiments found in the literature. The final \\ execution of this experiment and the correct obtaining of amplitude and \\ wavelength will contribute for the validation of the model here presented. \\ Keywords: roll waves, mudflows, hyperconcentrated fluids, light \\ absorption technique

\section{NOMENCLATURE} \\ C particles concentration on solution, ppm \\ $C^{*} \quad$ yield stress dimensionless variable \\ $\mathrm{Fr} \quad$ Froude number \\ $g$ gravity force, $\mathrm{m}^{2} / \mathrm{s}$ \\ $h$ flow height \\ $\mathrm{H} \quad$ flow height, $\mathrm{m}$ \\ $\hat{H} \quad$ infinitesimal disturbances on flow height $h$ \\ I Light intensity after crossing the path $h, \mathrm{~W}$ \\ $I_{0} \quad$ Emitted light intensity, $\mathrm{W}$ \\ $k$ disturbance wavenumber, $1 / \mathrm{m}$ \\ $m$ positive integer \\ $n \quad$ index flow \\ $t \quad$ time variable \\ $T \quad$ light transmittance \\ $u \quad$ mean velocity component on $x$ direction \\ $\mathrm{U}$ mean flow velocity to respect of $x, \mathrm{~m} / \mathrm{s}$ \\ $U$ wave propagation velocity \\ $\hat{U} \quad$ infinitesimal disturbance on mean velocity $u$ \\ $x \quad$ displacement variable \\ $\mathrm{Z}_{+} \quad$ integer positive set

\section{Greek symbols} \\ a momentum distribution coefficient \\ $\gamma \quad$ extinction coefficient, $1 / \mathrm{m}$ \\ $\Delta h \quad$ wave amplitude \\ $\varepsilon \quad$ molar absorptivity coefficient, $1 \cdot \mathrm{mol}^{-1} \mathrm{~cm}^{-1}$ \\ $\theta \quad$ steepness, degrees \\ $\lambda \quad$ wavelength of roll waves \\ $\rho$ fluid density, $\mathrm{kg} / \mathrm{m}^{3}$ \\ $\tau_{\mathrm{c}} \quad$ yield stress from Herschel-Bulkley rheological \\ model, $\mathrm{Pa}$ \\ $\omega$ disturbance frequency, $\mathrm{Hz}$

\section{Subscripts} \\ i imaginary part \\ $\mathrm{n}$ uniform flow \\ r real part \\ Observation: \\ All non-specified variables are dimensionless.
}




\section{INTRODUCTION}

Open channels flows under specific steepness may be favorable to the generation of periodic instabilities on the free surface, depending on fluid and flow characteristics. Usually found on artificial structures, such as spillways (Dressler, 1949), these waves may compromise the integrity of surrounding structures since they might lead to an intermittent flow. This configuration might result in occasional overflows, generation of debris flow, leaking of muddy and viscous materials, which can cause significant damage to nearby environment or structures. Such incidents are frequently exemplified through catastrophic landslides that occur after heavy rains in places with no vegetation cover. One can cite the cases of Angra dos Reis and Ilha da Madeira last year, and the events of Rio de Janeiro's north region and Minas Gerais on 2011, as examples of landslides that happened in places where the topography was very auspicious to the formation of roll waves. The picture shown in Fig. 1 shows the channel formed by the landslide of Angra dos Reis with a very steep slope. Furthermore, knowing that the fluid, composed by water from the rain and clay mixture from the soil, has non-Newtonian behavior, roll waves could have been formed and even amplified the damages as will be shown.

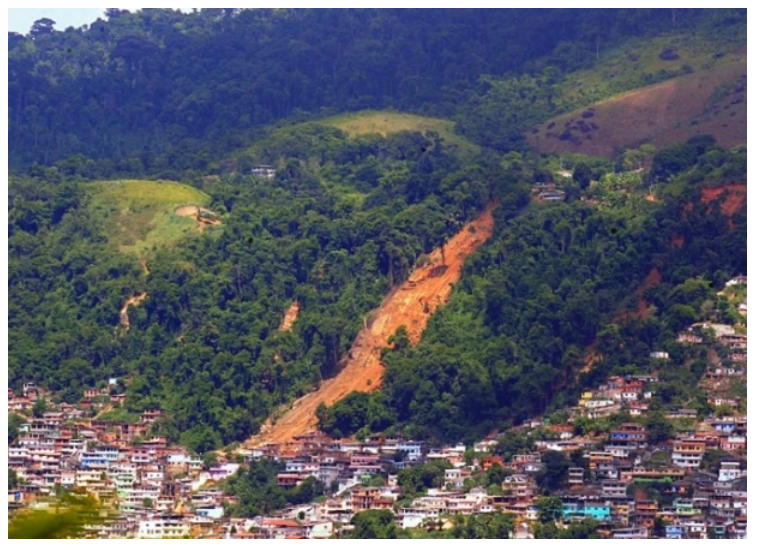

Figure 1. Carioca's cliff in Angra dos Reis, Rio de Janeiro, after the landslide of January, 2010.

Besides, this kind of waves can also appear on natural structures like rivers (Fer et al. 2002) and oceans (Swaters, 2003), and are even present in the human body, inside veins (Brook et al , 1999; Pedley, 1980), contributing for its research matter.

These typical waves, called roll waves, emerge in flows where the uniform flow, established by the balance between the body forces and the viscous forces, is disturbed by natural frequencies. In the flow early stage, a random oscillatory pattern will be noticed on the surface and, after a finite time, the regimen will be established and a periodic wave, usually a sawtooth-like, will characterize the instability visualized.
Many theories were developed in order to explain the generation of these waves, aiming to determine conditions for maintenance and generation of these waves. To represent the analytical models, numerical simulations are carried, which reproduce qualitatively the typical patterns on permanent regimen and also the conditions involved. Nevertheless, experimental procedures are necessary to validate and calibrate those models, which is not an easy task.

Several tests can be found in papers that present configurations for measuring systems and reasonable results, with good comparison analysis such as Kapitza (1948) and Liu et al. (1994). Although, they are usually implemented for Newtonian fluids, leaving the non-Newtonians behind. Coussot (1994) developed experiments with water-clay mixtures, considering his test-fluid as Herschel-Bulkley type, and was able to validate his roll wave stability criterion. However, no characteristics were measured by Coussot, and his observations can just be used to ensure the stability domain. Measures of wave amplitude and length are difficult to reach without interfering the flow: intrusive methods, such as conductive and capacitive, are quite easy to construct, operate and have a good precision, but they require the use of a probe that disturb the flow, what can be sometimes undesirable; non-intrusive ones don't interfere the flow, but the manipulation can compromise the rheological behavior adopted, or even limit measurements, thus hindering realistic values to be obtained.

The presence of these instabilities on mudflows and debris flows have been the research subject of the RMVP team (Rheology of Viscous and Viscoplastics Materials - FEIS/Unesp). The group develops analytical and numerical methods to predict physical properties of roll waves, and shows good results in agreement with those observed in the literature. The group now takes its efforts to research experimental methods to measure roll waves on open channels, as an attempt to fill the lack of data that exists in the literature and validate the theoretical models. If well accomplished, this work, still under development, will lead to the knowledge of the phenomenon's generation and spreading mechanisms, mainly on non-Newtonian fluids.

\section{MATHEMATICAL MODELING}

The modeling here presented was once developed by Ferreira (2007) based on the classic work of Dressler (1949), which falls upon discontinuous functions for roll waves. This mathematical work was carried through Cauchy's equations, inserting the Herschel-Bulkley rheological model (3 parameters) at the viscous part of the stress tensor. Saint-Venant hypothesis are considered. The system is vertically averaged and results in the following system, in dimensionless variables: 
Continuity equation:

$$
\frac{\partial h}{\partial t}+\frac{\partial(h u)}{\partial x}=0
$$

Momentum equation:

$$
\begin{gathered}
h\left(\frac{\partial u}{\partial t}+\alpha u \frac{\partial u}{\partial x}\right)-(\alpha-1) u \frac{\partial h}{\partial t}+\frac{1}{F r^{2}} \frac{\partial h}{\partial x}=h-C^{*}+ \\
-\left(1-C^{*}\right)\left[u h\left(\frac{1-C^{*}}{h-C^{*}}\right)\left(\frac{n+1+n C^{*}}{(n+1) h+n C^{*}}\right)\right]^{n}
\end{gathered}
$$

where $h$ is the fluid height, $u$, the mean flow velocity, $x$, longitudinal displacement over the channel, $t$, the temporal variable and $n$, the flow index, HerschelBulkley parameter. The velocity coefficient distribution, $\alpha$, is a function of fluid parameters:

$$
\alpha=\alpha\left(n, C^{*}\right)
$$

On the uniform flow, $(h, u)=\left(h_{\mathrm{n}}, u_{\mathrm{n}}\right)=(1,1)$ on the dimensionless system (Eq. (1) and (2)). The Froude number, $F r$, and the dimensionless coefficient for yield stress, $C^{*}$, are calculated from uniform regimen flow:

$$
\begin{gathered}
F r=\frac{\mathrm{U}_{\mathrm{n}}}{\sqrt{g \mathrm{H}_{\mathrm{n}} \cos \theta}} \\
C^{*}=\frac{\tau_{c}}{\rho g \mathrm{H}_{\mathrm{n}} \operatorname{sen} \theta}
\end{gathered}
$$

where $\rho$ is the fluid density, $g$, gravity acceleration, $\theta$, channel steepness, $H_{n}$ and $U_{n}$ are the fluid height and mean flow velocity in uniform flow. The yield stress from Herschel-Bulkley model, $\tau_{\mathrm{c}}$, is then represented by $C^{*}$. Through this system, it's possible to make an evaluation as a dynamical system and then stability analysis can be done.

\section{Linear Stability Analysis}

Linear stability theory is a powerful tool that allows observation of important information from the dynamical system, such as growth rate and propagation velocity of instabilities. Through this method, widely discussed by Briggs (1964) and Huerre and Monkewitz (1990), it is possible to detail the necessary conditions for instabilities formation on the system. Di Cristo (2005) carried this theory to evaluate the convective nature of roll waves instabilities, i.e. demonstrate how waves can appear and grow in time and space if flow conditions are favorable. The objective is reach the dispersion equation for the system and analyze how an infinitesimal perturbation behave.
Infinitesimal disturbances, $\hat{H}(x, t)$ and $\hat{U}(x, t)$, are added into the system in uniform regimen, Eq. (1) and (2), meaning that:

$$
\begin{aligned}
& h=1+\hat{H}(x, t) \\
& u=1+\hat{U}(x, t)
\end{aligned}
$$

with $|\hat{H}|,|\hat{U}|<<1$. The system is now represented as:

$$
\frac{\partial \hat{H}}{\partial t}+\frac{\partial \hat{H}}{\partial x}+\frac{\partial \hat{U}}{\partial x}=0
$$

$$
\begin{gathered}
\frac{\partial \hat{U}}{\partial t}+\alpha \frac{\partial \hat{U}}{\partial x}-(\alpha-1) \frac{\partial \hat{H}}{\partial t}+\frac{1}{F r^{2}} \frac{\partial \hat{H}}{\partial x}=1+\hat{H}-C^{*}+ \\
-\left(1-C^{*}\right)\left[(1+\hat{U})(1+\hat{H})\left(\frac{1-C^{*}}{1+\hat{H}-C^{*}}\right) .\right. \\
\left.\cdot\left(\frac{n+1+n C^{*}}{(n+1)(1+\hat{H})+n C^{*}}\right)\right]^{n}
\end{gathered}
$$

Solving the above system (Eq. (7) and (8)) for the variation of height, $\hat{H}(x, t)$, the following partial differential equation is found:

$$
\begin{aligned}
\frac{\partial^{2} \hat{H}}{\partial t^{2}}+\left(1+\frac{1}{F r^{2}}\right) \frac{\partial^{2} \hat{H}}{\partial x^{2}}+2 \alpha \frac{\partial^{2} \hat{H}}{\partial x \partial t}+ & \\
& n\left(1-C^{*}\right) \frac{\partial \hat{H}}{\partial t}+\frac{(n+1)(2 n+1)}{\left(n+1++n C^{*}\right)} \frac{\partial \hat{H}}{\partial x}=0
\end{aligned}
$$

From the linear theory, the surface wave problem is given by the solution of Laplace equation through the separation of variables method, applying the kinematic boundary conditions of the problem at the bottom and at the surface. On the other hand, knowing the solution periodicity and uniformity, one can consider the perturbation of the type:

$$
\hat{H}=|\hat{H}| e^{i(\omega t-k x)}
$$

where $|\hat{H}|$ is a magnitude value (constant), $k$, wave number, and $\omega$, the disturbance frequency; $i$ represents the imaginary number.

A wave is defined as unstable if there is a complex frequency $\omega=\omega_{\mathrm{r}}+i \omega_{\mathrm{i}}$, with $\omega_{\mathrm{i}}<0$, for a real wavenumber $k$, obtained from the dispersion equation of the system, meaning that there is temporal growth of infinitesimal spatial disturbances. The group of parameters $\left(n, F r, C^{*}\right)$ consists of the control parameters of the problem. To simplify the manipulation of dispersion relation, two groups of parameters will be adjusted:

$$
\vartheta\left(n, C^{*}\right)=n\left(1-C^{*}\right)
$$




$$
\varphi\left(n, C^{*}\right)=\frac{(n+1)(2 n+1)}{\left(n+1+n C^{*}\right)}
$$

Thus, manipulating Eq. (9) and (10), inserting Eq. (11) and (12), the following dispersion equation is found:

$$
\begin{gathered}
D\left(\omega, k, n, F r, C^{*}\right)=0 \\
\omega^{2}+\left(\alpha-\frac{1}{F r^{2}}\right) k^{2}-2 \alpha k \omega-i \vartheta \omega+i \varphi k=0
\end{gathered}
$$

Solving Eq. (14) for $\omega$ and considering $\omega_{\mathrm{i}}<0$ (favorable temporal growth rate), one can reach a criterion that specify a proper domain for generating instabilities to respect of Froude number:

$$
F r>F r_{\min }=\frac{\vartheta}{\sqrt{\varphi^{2}-2 \alpha \varphi \vartheta+\alpha \vartheta^{2}}}
$$

\section{First-order equation for Roll waves generation}

The model here presented was developed by Ferreira (2007), member of RMVP/Unesp team, and uses the continuity and momentum equations, Eq. (1) and (2), to elaborate one single differential equation that represents the phenomenon. The system is transferred to a mobile coordinate system and manipulated to reach a first-order differential equation (all mathematical steps are detailed in Ferreira (2007)):

$$
\frac{\partial h}{\partial x} \equiv \frac{F(h)}{G(h)}
$$

where

$$
\begin{gathered}
F(h)=h-C^{*}-\left(1-C^{*}\right)[((h-1) U+1) \cdot \\
\left.\cdot \frac{\left(1-C^{*}\right)\left(n+1+n C^{*}\right)}{\left(h-C^{*}\right)\left((n+1) h+n C^{*}\right)}\right]^{n} \\
G(h)=\frac{h}{F r^{2}}+(\alpha-1) U^{2}-\frac{\alpha(U-1)^{2}}{h^{2}}
\end{gathered}
$$

The wave celerity, $U$ is function of fluid and flow parameters:

$$
U=U(\alpha, F r)
$$

However, since this is a first-order model, it is prone to specifics peculiarities that must be treated, as well as the classic work of Dressler (1949). As the construction of the equation solution arises, a discontinuity appears, exactly where $h=h_{\mathrm{n}}$, causing a indetermination, $F(h) / G(h)=0 / 0$. At that point, Rankine-Hugoniot's shock conditions are called to keep continuity in passing through that very point, as long as it is known that exists a continuous solution. Once it's done, the continuous solution is given by:

$$
h(x)=\int_{m \lambda}^{(m+1) \lambda} \frac{F(h)}{G(h)} d x, \quad \text { for } m \in Z_{+}
$$

bounded by the wave length $\lambda$, the interval between two successive shocks:

$$
\lambda=\int_{h_{1}}^{h_{2}\left(h_{1}\right)} \frac{G(h)}{F(h)} d h
$$

$$
\begin{gathered}
h_{2}=\left[\left(\frac{h_{1}}{2}+(\alpha-1) U^{2} F r^{2}\right)^{2}+\frac{2 \alpha(1-U)^{2} F r^{2}}{h_{1}}\right]^{\frac{1}{2}}+ \\
-\frac{h_{1}}{2}-(\alpha-1) U^{2} F r^{2}
\end{gathered}
$$

The value of $h_{2}$ is calculated through the Eq. (21), while $h_{1}$ must be estimated for real values of $F(h) / G(h)$. The procedure adopted here was to impose $h_{1} 1 \%$ greater than the minimum value for $h$ so $F(h) / G(h)$ is real.

Schematically, the phenomenon can be represented by the following Fig. 2:

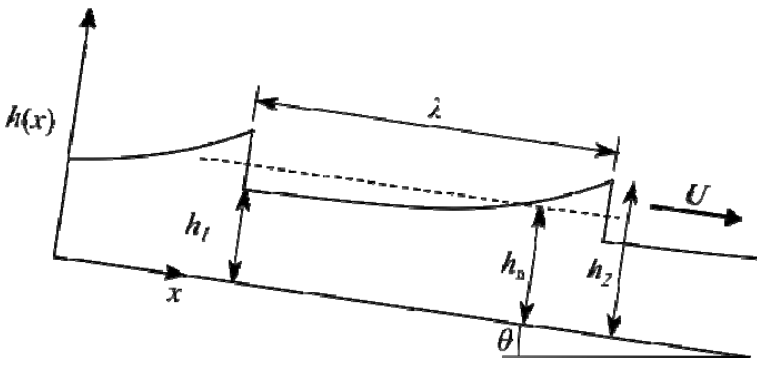

Figure 2. Sketch representing 1D roll waves falling down open channel and its typical dimensions.

\section{Representativeness Analysis}

In order to prove the representativeness of the model presented is last section, numerical simulations were done by testing the rheology of the fluid. In this section, Matlab/Simulink tool was employed, since it is an excellent environment for dynamical systems and has a large library of function.

For Newtonian fluids, the fluid flow starts to shear with the minimal force applied to it so the dimensionless coefficient for critical stress is minimal $\left(C^{*}=0\right)$ and, how the shearing stress dependence on the deformation is linear, the index flow must be 1 ( $n$ $=1$ ). This type of fluid represents common fluids usually observed in nature, such as water and air. Roll wave pattern may arise on this configuration and it is expected that the model would be capable of generating that pattern. Then, for $F r=1\left(F r>F r_{\text {min }}\right)$, 
roll waves were numerically generated as Fig. 3 shows.

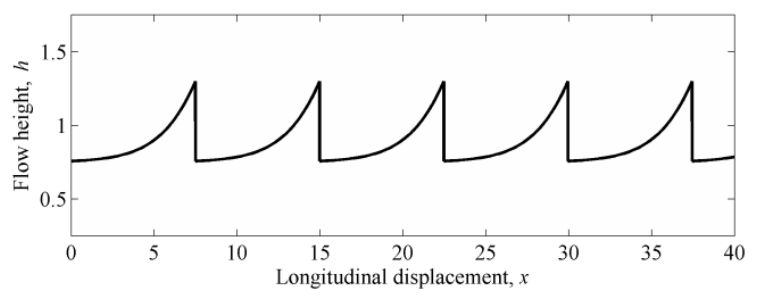

Figure 3. Numerical simulation of roll waves in

Newtonian fluids $\left(n=1 ; C^{*}=0 ; F r=1\right)$. Wave celerity $U_{1}=2,31$, wave amplitude $\Delta h_{1}=0,542$, and wavelength $\lambda_{1}=7,49$.

In a comparison between these results and those found by Dressler (1949), it's clear that the first-order model developed by the team was capable of reproducing qualitatively roll waves in open channels flow of Newtonian fluids. However, as said before, the proposal was to generate waves for nonNewtonian fluids flow due to their presence on important natural phenomena, such as landslides, avalanches, etc.

For a power-law fluid, with $n=0,75$, the numerical model was simulated and the result is shown at Fig. 4.

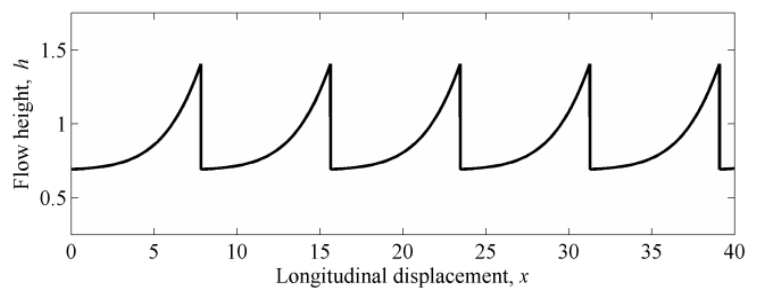

Figure 4. Numerical simulation of roll waves in power-law fluid $\left(n=0,75 ; C^{*}=0 ; F r=1\right)$. Wave celerity $U_{2}=2,28$, wave amplitude $\Delta h_{2}=0,714$, and wavelength $\lambda_{2}=7,82$.

A simple analysis based on the results can show some characteristics of the roll-wave phenomenon. The non-Newtonian effect of the index flow contribute to the "enlargement" of the wave, increasing the amplitude $\left(\Delta h_{2}>\Delta h_{1}\right)$ and the wavelength $\left(\lambda_{2}>\lambda_{1}\right)(\mathrm{Ng}$ and Mei, 1994). Meanwhile, the velocity propagation is decreased $\left(U_{2}<U_{1}\right)$. Adding complexity to the rheological behavior, the Herschel-Bulkley model for fluids is reached. Besides the non-linear relation between the shearing stress and the deformation, the presence of the yield stress implies that the body forces need to surpass a threshold value so the fluid can begin its movement. This effect is summarized by the yield stress dimensionless coefficient $C^{*}$. Adopting $C^{*}=0,3$, numerical simulation was carried (Fig. 5).

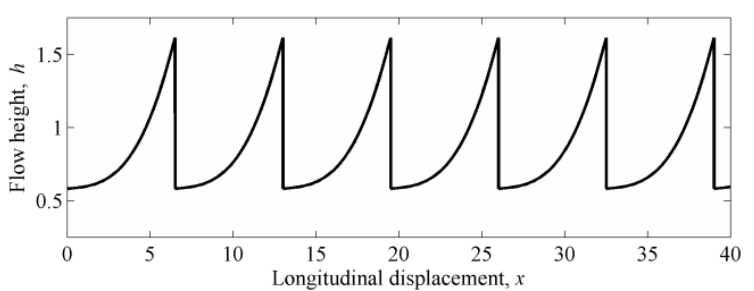

Figure 5. Numerical simulation of roll waves in Herschel-Bulkley fluid ( $\left.n=0,75 ; C^{*}=0,3 ; F r=1\right)$. Wave celerity $U_{3}=2,20$, wave amplitude $\Delta h_{3}=$ 1,030 , and wavelength $\lambda_{3}=6,50$.

The coherence effect due to the fluid rheological behavior contributes to the increase of the wave amplitude $\left(\Delta h_{3}>\Delta h_{2}\right)$ and to the decrease of the wavelength $\left(\lambda_{3}<\lambda_{2}\right)$. Also, one more time the propagation velocity of the waves is decreased $\left(U_{3}<\right.$ $U_{2}$ ). The last effect is equivalent to the anticipation of the wave's generation reported by Maciel et al. (1997) when increasing the coherence effect in his secondorder model for roll waves in Bingham fluids.

These effects have been well explored mathematically and numerically by many researchers all over the world and the results found by RMVP team show good agreement with them. As will be shown, the work that is now being developed is related to the physical evaluation of these waves, in a attempt to explore quantitatively the models developed and make them technically usable.

\section{EXPERIMENTAL PROCEEDINGS}

The first-order model presented before can be used to represent many fluids with different rheological behavior, since the model inserted has 3 parameters that can express parameters from others models, such as Bingham, Power-law, and even Newtonian. From this model, numerical methods were exploited to verify how and when instabilities appears in open channel flows using many environments, like Python, Fortran and Matlab/Simulink. The programs developed were able to generate wave-like patterns just like presumed by the analytical modeling, but to assume that the data acquired from numerical simulations are realistic, it is necessary to validate the numerical and analytical models adopted. At this point the major problem emerges, where experimental data for roll waves has to be acquired by empirical tests.

Taking measurements of amplitude and wavelength are delicate. If it's necessary to make it while disturbing the flow it can be done by inserting resistive probes or capacitive systems, which are easily manipulated, but may contribute to misevaluate the data acquired, besides having no good precision. In other hand, non-intrusive systems, such as laser-based, have much better precision but are more complicated to handle. An option has to be made depending on how it is desired the flow to be: if 
large and not so fine, intrusive system could be an easy and cheap choice; for sensitive and delicate flows, non-intrusive are a wiser option, and must be carefully studied for adapting to the flow configuration.

The experimentation in roll waves initialized with Cornish (1934) that registered them for the first time and described their behavior in prismatic and artificial channels. After that, the experiments carried by Brock (1969) were very useful to comprehend in what circumstances those waves would appear (Fig. 6). However, the apparatus used, pressure transducers and resistive probe (called by him as wire gage), had low precision and were intrusive to the flow.

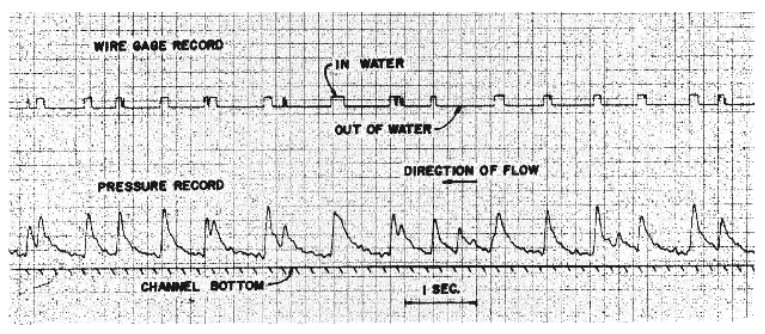

Figure 6. Results obtained by Brock (1969) on his experimental apparatus for measuring roll waves on turbulent flow. (Adapted from Ph.D. thesis of Richard R. Brock, California Institute of Technology).

Later, one of the most impressive works found, an apparatus composed by a channel capable of resist to external perturbations were developed by the research of Gollub (Liu et al. (1994); Liu et al. 1993). The measures were taken by fluorescence imaging technique which consists in adding a fluorescent dye to the fluid and illuminate the flow with a special light, which gives the flow his own bright. Assisted by a camera, the flow height can be recorded in images that, after treated, will have the wave data, as shown in Fig. 7.

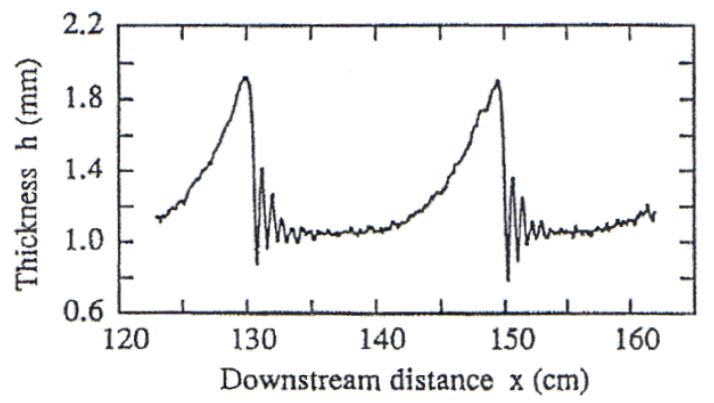

Figure 7. Profile of a wave train obtained from a disturbed uniform flow; $\omega=1.5 \mathrm{~Hz}$ (disturbing frequency), $R e=29$ (Reynolds number) and $6.4^{\circ}$ of steepness $(\theta)$. Adapted from Liu et al. (1994).

Recently, light-based techniques have been used to get more reliable and precise data for different flow configurations, such as total external or internal reflection, light absorption, interferometry methods. Mouza et al. (1999) were capable of acquiring a precise data, compared with a conductive probe, for a uniform flow of a thin layer fluid (Fig. 8). Thin layer fluid, or film flow, is a phenomenon well-explored by chemists, physicists and engineers due to its presence in many industrial process such as electrochemical plating, crystal growth, condensers, etc. The measurement techniques adopted by these experts for measuring film flows are useful because they are quite precise and have a fast time response. Moreover, instabilities can be observed in film flows and even roll wave-type (Fig. 7).

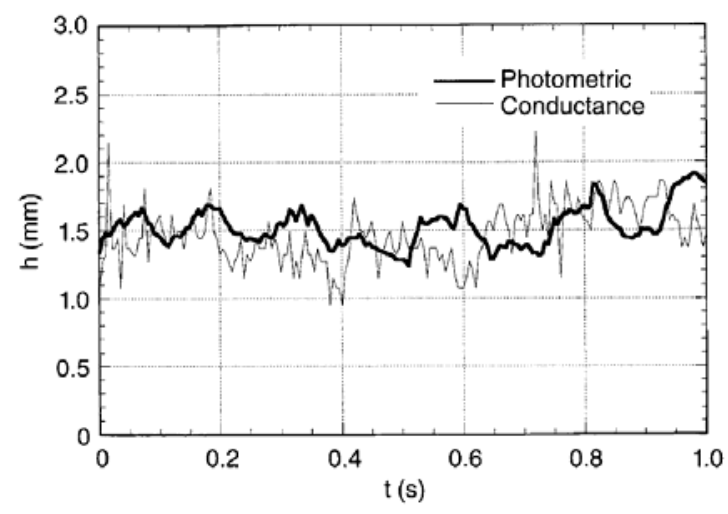

Figure 8. Measure from a film fluid (water + methylene blue solution) obtained by photometric system (dark line) and by conductive probe (light line), for the same flow configuration. Adapted from Mouza et al. (1999).

When measuring film flows by non-intrusive systems, many photometrics methods are able to acquire information on flow height, but the choose depends on how precise the measurements need to be and how difficult (and expensive) will it be. In the next section, the method adopted by the group is described.

\section{Photometric Measurement System: Light Absorption Technique}

Whenever light passes through any medium (solid, liquid or gas), the electromagnetic waves that composes it will suffer from interference, scattering or being absorbed, by the medium. Also, depending on how the light focus the medium it will reflect and refract at some degree. If, for example, a light beam is orthogonally directed through a liquid sample, from a solution in a concentration $\mathrm{C}$, and aligned with a photodetector (positioned after the sample) the light intensity perceived by the detector is proportional to the concentration of the substance in solution and to the height of that liquid, obeying to the BeerLambert's Law:

$$
I=I_{0} e^{-\gamma h}=I_{0} e^{-\varepsilon C h}
$$


where $I_{0}$ is the incident light intensity, $\varepsilon$ is the molar absorptivity of the substance and $h$ is the pathlength of the light (flow height). No light scattering is considered. Then, the transmittance, denoted by $T$, will be

$$
T \equiv \frac{I}{I_{0}}=e^{-\varepsilon C h}
$$

and the absorbance, $A$ :

$$
A \equiv-\log \left(\frac{I}{I_{0}}\right)=\varepsilon C h=\gamma h
$$

which express a linear relation between the quantity of light absorbed and the light pathlength.

Some precise results for film flows can be found in the literature. Barter et al. (1993) were able to get height and slope information from a wave pattern generated inside a water tank with an pneumatic oscillator. The certainty reported is about $0.005 \mathrm{~mm}$ which is a very precise measurement for fluid height.

Some difficulties appears when trying to develop this experiment which lays on how will light reflection and refraction disturb the final result. Most of the tests are made considering the flow as uniform, maintaining it undisturbed from undesired sources. The uniform flow allows the apparatus' user to keep approximately $90^{\circ}$ angle of the incident light, minimizing the effects of the reflection. Refraction effect do not bother as much as reflection and a wellchosen photodetector, with a wide effective area, will minimize its effects.

\section{Experimental results}

Aware of the studies here referenced, our team initiated the project of a calibration set for a photometric system following the work of Mouza et al. (1999). A BPX65 photodetector was coupled with a He-Ne laser, $633 \mathrm{~nm}$, to mount the elementary part of the system. Three different solutions were elaborated using pure water and methylene blue in different concentrations.

The first results for calibration tests exhibited at Fig. 9 shows good linearity response of the apparatus but not so good correlation. The calibration coefficient found, that correspond to the molar absorptivity coefficient of the methylene blue, had a value that does not fit the data acquired very well.

Figure 10 shows that a major problem exists for low heights of the sample.

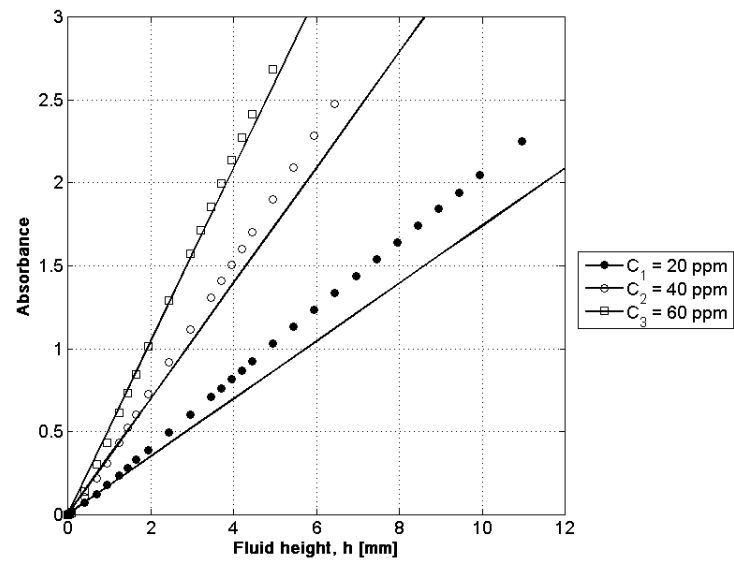

Figure 9. Results for calibration tests for pure water + methylene blue from solutions in different concentrations.

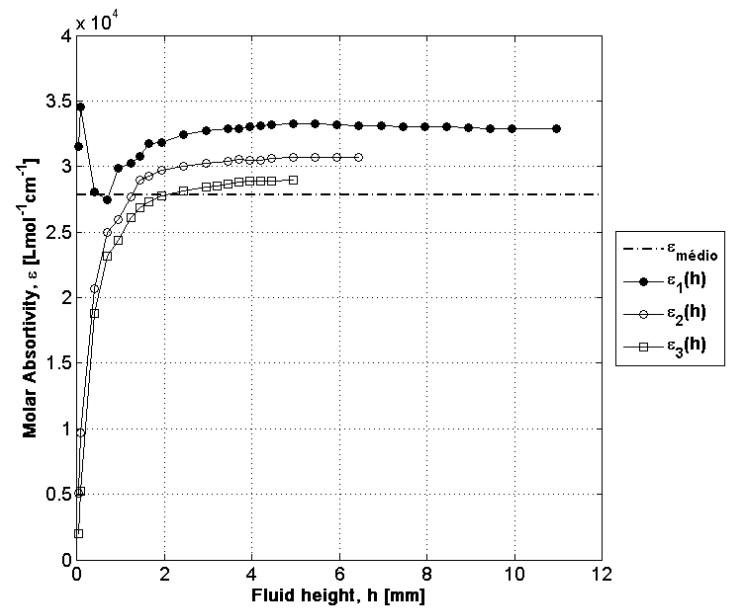

Figure 10. Molar absorptivity, $\varepsilon$, plotted against fluid height, $h$. The indicial number refers to respective concentration (1, 2 and 3$)$.

Below $2 \mathrm{~mm}$ of liquid height, for all 3 tests, the molar absorptivity had a non-constant behavior what suggests that the substance diluted, methylene blue, had it natural characteristics changed with the fluid height, which it is not true. Statistical analysis showed a mean error of $15,57 \%$ for the system, which is a considerable value.

It is evident that the mean value found, $\varepsilon_{\text {medio }}=$ $2.78 \cdot 10^{4} 1 \cdot \mathrm{mol}^{-1} \mathrm{~cm}^{-1}$, is lower than the mean values of each test because of the low values for the absorptivity below $2 \mathrm{~mm}$. Moreover, tabulated values for $\varepsilon$ to a $633 \mathrm{~nm}$ light source are between $4.29 \cdot 10^{4}$ and $4.42 \cdot 10^{4} 1 \cdot \mathrm{mol}^{-1} \mathrm{~cm}^{-1}$ (data obtained from Oregon Medical Laser Center, on February, 15th, 2011 at http://omlc.ogi.edu/spectra/mb/mb-water.html). Test were carried at the laboratory using a spectrofotometric equipment DR 5000 UV/VIS at wavelength $633 \mathrm{~nm}$ and showed that the molar absorptivity of the methylene blue is 
$4.37 \cdot 10^{4} 1 \cdot \mathrm{mol}^{-1} \mathrm{~cm}^{-1}$ which is between the range specified by the reference.

\section{CONCLUSIONS}

Roll wave shows discontinuities that must be taken seriously and with caution when developing effective models and realistic representation of the phenomenon. However, based on solid theories and established models, the mathematical model presented by the team shows itself as congruent as the classic ones. The results acquired by the numerical modeling through Matlab/Simulink, an effective tool for solving dynamical systems, illustrated the results expected with fidelity and assured models such as $\mathrm{Ng}$ and Mei (1994), Maciel et al. (1997) and Dressler (1949). At this point, it's relevant to note that the $1 \mathrm{D}$ mathematical model here presented can generate roll-waves solutions in a few seconds for a specific parameters' group.

A brief conceptualization about the experimental method adopted by team were made based on well-known experiments in fluids flow. The initial attempt to measure thin layer fluid was acceptable showing good behavior of the system, in agreement with the light absorption theory. In order to reduce the error detected, more tests should be conducted, intensifying the efforts to minimize the random errors of the system and making easier to detect systematic errors that could be infecting the tests.

With the calibration apparatus complete and functional, its adaptation to the channel will be made, allowing the employment of the photometric system to measure film flows in motion and with oscillatory pattern.

The data acquired will make possible the validation of the numerical and mathematical methods adopted for estimating roll waves formation in Newtonian and non-Newtonian fluids flow at open channels. It will also allow the prediction of the circumstances where roll waves could contribute to intensify natural hazards of such nature and its risks.

\section{ACKNOWLEDGMENTS}

Our thanks to FAPESP, CNPq and CAPES, for project funding and scholarships.

\section{REFERENCES}

Barter, J.D., Beach, K.L. and Lee, P.H.Y., 1993, Collocated and simultaneous measurement of surface slope and amplitude of water waves, Review of Scientific Instruments, Vol. 64, No. 9, pp. 26612665.

Briggs, R.J., 1964. Electron-stream interaction with plasmas, MIT Press.
Brock, R.R., 1969, Development of roll-wave trains in open channels, Journal of Hydraulics Division, Vol. 95, pp. 1401-1427.

Brook, B.S., Falle, S.A.E.G. and Pedley, T.J., 1999, Numerical solutions for unsteady gravitydriven flows in collapsible tubes: evolution and rollwave instability of a steady state, Journal of Fluid Mechanics, Vol. 396, pp. 223-256.

Cornish, V., 1934. Ocean waves and Kindred Geophysical phenomena, Cambridge University Press.

Coussot, P., 1994, Steady, laminar, flow of concentrated mud suspensions in open channel, Journal of Hydraulic Engineering, Vol. 32, No. 4, pp. 535-559.

Di Cristo, C. and Vacca, A., 2005, On the convective nature of roll waves instability, Journal Applied Mathematics, Vol. 3, pp. 259-271.

Dressler, R. F., 1949, Mathematical solution of the problem of roll waves in inclined open channels, Communs Pure Applied Mathematics, Vol. 2, pp. 149-194.

Fer, I., Lemmin, U. and Thorpe, S.A., 2002, Winter cascading of cold water in lake Geneva, Journal of Geophysical Research, Vol. 17, No. C6, pp. 1-16.

Ferreira, F. O., 2007, Abordagem matemática de roll waves em escoamentos hiperconcentrados com superfície livre, Master Dissertation, Faculdade de Engenharia de Ilha Solteira - Universidade Estadual Paulista, Ilha Solteira, São Paulo, Brazil. (in Portuguese)

Huerre, P. and Monkewitz, P.A., 1990, Local and global instabilities in spatially developing flows, Annual Review of Fluid Mechanics, Vol. 22, pp. 473537.

Kapitza, P.L., 1948, Wave flow of thin layers of a viscous fluid - Collected Papers of P.L. Kapitza, Vol. II. Pergamon Press (1965).

Liu, J. and Gollub, J.P., 1994, Solitary wave dynamics of film flows, Physics of Fluids, Vol. 6, pp. 1702-1712.

Liu, J., Paul, J.D. and Gollub, J.P., 1993, Measurement of the primary instabilities of film flows, J. of Fluid Mechanics, Vol. 250, pp. 69-101.

Maciel, G.F., Vila, J.P. and Martinet, G., 1997, Roll wave formation in the non-Newtonian flows, In XIV COBEM, Bauru, SP, Brazil.

Mouza, A.A., Vlachos, N.A., Paras, S.V. and Karabelas, A.J., 1999, Measurement of liquid film thickness using a laser light absorption method, Experiments in Fluids, Vol. 28, pp. 355-359.

$\mathrm{Ng}$, C. and Mei, C.C., 1994, Roll waves on a shallow layer of mud modelled as a power-law fluid, Journal of Fluid Mechanics, Vol. 263, pp. 151-183.

Pedley, T.J., 1980, Fluid mechanics of large blood vessels, Cambridge University Press.

Swaters, G.E., 2003, Baroclinic characteristics of frictionally destabilized abyssal overflows, Journal of Fluid Mechanics, Vol. 489, pp. 349-379.

Received: July 30, 2011

Revised: August 30, 201

Accepted: September 30, 2011 